Article

\title{
Synthesis and SAR Study of Novel Peptide Aldehydes as Inhibitors of 20S Proteasome
}

Yuheng Ma, Bo Xu, Yuan Fang, Zhenjun Yang, Jingrong Cui, Liangren Zhang * and Lihe Zhang

State Key Laboratory of Natural and Biomimetic Drugs, School of Pharmaceutical Sciences, Peking University, Beijing 100191, China

* Author to whom correspondence should be addressed; E-Mail: liangren@bjmu.edu.cn; Tel.: +86-10-82802567; Fax: +86-10-82805063.

Received: 1 August 2011; in revised form: 29 August 2011 / Accepted: 30 August 2011 / Published: 5 September 2011

\begin{abstract}
Based on the analysis of the crystal structure of MG101 (1) and 20S proteasomes, a new series of peptide aldehyde derivatives were designed and synthesized. Their ability to inhibit $20 \mathrm{~S}$ proteasome was assayed. Among them, Cbz-Glu(OtBu)-PheLeucinal (3c), Cbz-Glu(OtBu)-Leu-Leucinal (3d), and Boc-Ser(OBzl)-Leu-Leucinal (3o) exhibited the most activity, which represented an order of magnitude enhancement compared with MG132 (2). The covalent docking protocol was used to explore the binding mode. The structure-activity relationship of the peptide aldehyde inhibitors is discussed.
\end{abstract}

Keywords: 20S proteasome; inhibitor; peptide aldehydes; synthesis; structure-activity relationship

\section{Introduction}

Lysosomes and the ubiquitin-proteasome pathway (UPP) are two major routes for cellular protein degradation [1-3]. The UPP is essential for many cellular regulatory mechanisms and plays a crucial role in the regulation of many physiological processes. For example, degradation of the p53 tumor suppressors [4] and inhibition of cyclin-dependent p27 kinases [5] can promote tumorigenesis, disorders of protein degradation that originated from UPP can cause the development of many human diseases, such as cancer, Alzheimer's and Parkinson's diseases, etc. [6-8]. Recently, the study of 
proteasome inhibition has received much attention [9-14]. In UPP, proteolysis takes place in the 26S proteasome, which consist of one or two 19S regulatory particles (RP) [15] and a central catalytic particle known as the $20 \mathrm{~S}$ proteasome (CP). The $20 \mathrm{~S}$ proteasome is a large cylindrically-shaped complex composed of two copies of seven distinct $\alpha$ - and seven distinct $\beta$-type subunits [16,17]. It possesses three protease activities, namely the post-glutamyl-peptide hydrolyzing (PGPH), the trypsinlike (T-L), and the chymotrypsin-like (ChT-L) activity, which are assigned as the active subunits $\beta 1$, $\beta 2$, and $\beta 5$, respectively $[18,19]$.

Small molecules, have been developed to inhibit the proteasome such as cyclic peptides [10,20-22], peptide boric acids [23], peptide epoxides [24], peptide vinyl sulfones [25], and nonpeptidic molecules [26-29]. Among all the proteasome inhibitors ever studied, peptide aldehydes were the first developed and are still the most widely used in in vitro and in vivo studies [30]. MG101 (1, Ac-Leu-Leu- $n$ Leu-al, Figure 1), one of calpain inhibitors, is the first well-known 20S proteasome inhibitor [16,17,31]. The crystal structure of the $20 \mathrm{~S}$ proteasome in complex with MG101 confirms that the hydroxyl group of the N-terminal threonine of the $\beta 5$ subunit reacted with the aldehyde group and formed a reversible hemiacetal. MG132 (2, Cbz-Leu-Leu-Leu-Al, Figure 1), a more potent and selective analog of MG101, which bears a benzyloxycarbonyl group instead of an acetyl group, is one of the most commonly used synthetic proteasome inhibitors [32,33]. Up to now, many peptide aldehydes have been designed and synthesized [34,35].

Figure 1. Structures of MG101 (1), MG132 (2) and peptide aldehydes (3).

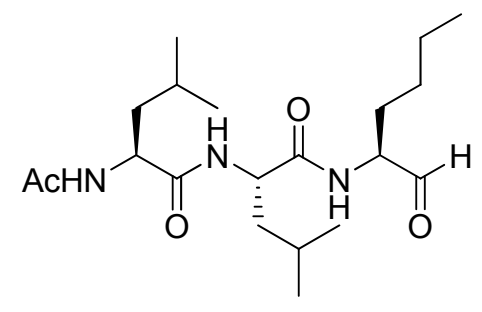

1 MG101<smiles>CC(C)CC(NC(=O)COC(C)C)C(=O)NC(CC(C)C)C(=O)NC(C)C</smiles>

2 MG132

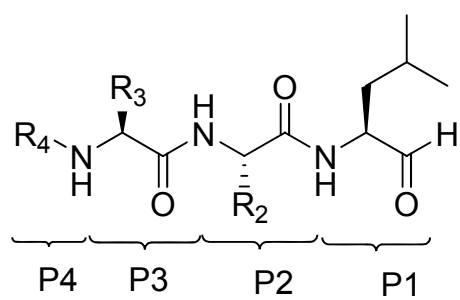

3 R=substituted groups $\mathrm{P}=$ substituted amino acids

Previous studies have demonstrated that hydrophobic groups around the P1 and P3 positions are beneficial to enhance the activity of peptide aldehydes 3 [36-38]. Bulky substituents at the P2 position and aromatic groups at $\mathrm{P} 4$ position also contribute to enhance the inhibitory activity [37]. According to the crystal structure of complexed MG101 and 20S proteasome, the leucine side chain of P3 projects into the $\mathrm{S} 3$ pocket of $\beta 5$ subunit, which is an open space in the vicinity of the isopropyl groups. Since the P3-leucine moiety only partially fills the S3 pocket, we supposed that introducing a large group at P3 to fill the open space might enhance inhibitory activity. Thus, in this study, we mainly focus on the variation of P3 position to reveal the structure-activity relationships. A series of peptide aldehyde derivatives are designed which have a bulky P3 moiety aiming to increase the hydrophobic interactions with S3. 


\section{Results and Discussion}

\subsection{Synthesis of Peptide Aldehydes 3a-r}

The synthesis of the peptide aldehydes is shown in Scheme 1. L-Leucine (4) was treated with $\mathrm{NaBH}_{4}$ and $\mathrm{I}_{2}$ under argon to give L-leucinol (5) in $89 \%$ yield [39], which was then coupled with Boc-protected amino acids to form the dipeptide alcohols 6 in $71 \%-80 \%$ yield. The dipeptide alcohold were deprotected with $20 \%$ trifluoroacetic acid in dichloromethane, followed by reaction with $t$-butoxy-carbonyl (Boc)- or benzyloxycarbonyl (Cbz)-protected amino acids to give 8a-r (crude products were used in the next step without further purification). After Swern oxidation [40], compounds 3a-r were obtained in $49 \%-59 \%$ yields.

Scheme 1. Synthesis of peptide aldehydes 3.
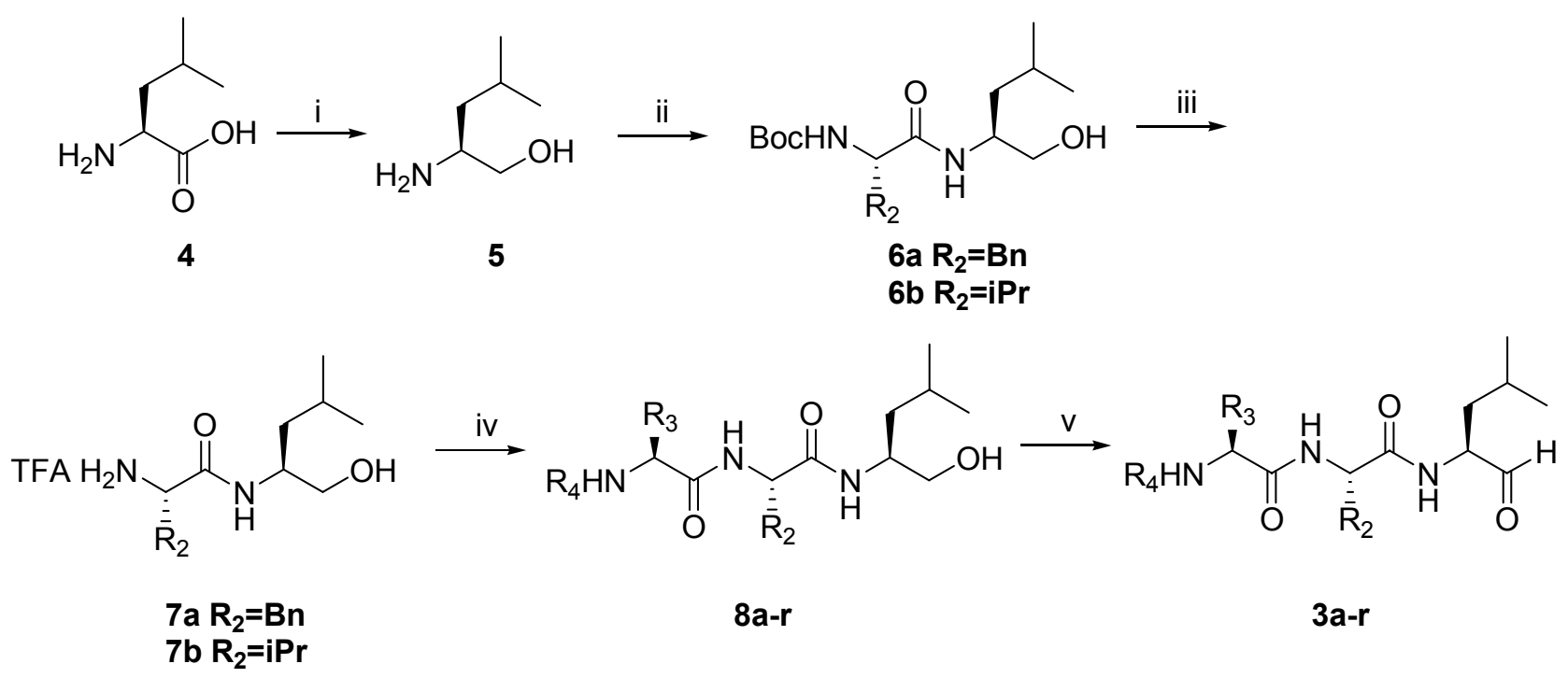

Reagents and Conditions: (i) $\mathrm{NaBH}_{4}, \mathrm{I}_{2}, 89 \%$; (ii) Boc-Leu-OH or Boc-Phe-OH, DCC, HOBt, $0{ }^{\circ} \mathrm{C}$ to rt, 76\%; (iii) TFA/DCM; (iv) Cbz-AA-OH or Boc-AA-OH, DCC, HOBt, DIPEA, $0{ }^{\circ} \mathrm{C}$ to rt; (v) oxalyl chloride, DMSO, TEA, $-78^{\circ} \mathrm{C}, 49 \%-58 \%$.

\subsection{Assays for Proteasome Activities and SAR}

Inhibitory activities of peptide aldehydes on the $20 \mathrm{~S}$ proteasome are assayed in vitro [41]. MG132 was used as the positive control (Table 1). The results indicate that most of the peptide aldehydes exhibited inhibitory activities against ChT-L, which is closely associated with the substituted amino acids at P3. Out of 17 synthesized compounds, nine exhibited inhibitory activities with $\mathrm{IC}_{50}$ in the $n \mathrm{M}$ range, and three compounds in particular (3c, 3d, and 3o) demonstrated much higher activities than the control MG132.

These inhibitors can be classified into $\mathrm{Cbz}$ and Boc series, based on the moiety at the P4 position $\left(\mathrm{R}_{4}\right)$. Among the Cbz series, a P3 residue with a bulky hydrophobic branch (compounds 3a-3e, 3h) affords a highly active inhibitor, whereas, the electropositive branches (compounds $\mathbf{3 f}$ and $\mathbf{3 g}$ ) show dramatically decreased activity. Compounds $\mathbf{3 c}$ and $\mathbf{3 d}$ exhibited about 10-fold higher activity than the others, indicating that a relatively long bulky side chain may favor the increase of activity. For the Boc 
series, the P3 residue with a hydrophobic bulky side chain generally affords an active ChT-L inhibitor. The activities of Boc series compounds with different P3 residues go in an order of $\mathbf{3 o}>\mathbf{3 p}>\mathbf{3 i}$ and $\mathbf{3 k}>\mathbf{3 j}>\mathbf{3 l}$, also showing that a bulky side chain is too long to give higher activity. Both $\mathbf{3 m}$ and $\mathbf{3 n}$ show poor activities with $\mathrm{IC}_{50}>50 \mu \mathrm{M}$, and the reason for this deceased potency might be derived from the presence of a proline pyrrolidine moiety at the P3 position, which is consistent with reported results [37]. Furthermore, in contrast to the Cbz series, in which the activity does not vary obviously with the side chain of P2, in the Boc series, a benzyl group at P2 (3i and 3k) affords much higher activity than the corresponding isobutyl branched compound ( $\mathbf{3} \mathbf{j}$ and $\mathbf{3 1})$.

Table 1. Inhibition of peptide aldehydes to ChT-L activity of 20S proteasome.

\begin{tabular}{|c|c|c|c|c|}
\hline Compounds & R4 & P3 & $\mathbf{P 2}$ & ChT-L $\left(\mathrm{IC}_{50}, \mu \mathrm{M}\right)$ \\
\hline $\mathbf{3 a}$ & $\mathrm{Cbz}$ & $\mathrm{Asp}\left(\mathrm{O}^{t} \mathrm{Bu}\right)$ & Phe & $0.21 \pm 0.014$ \\
\hline $\mathbf{3 b}$ & $\mathrm{Cbz}$ & $\operatorname{Asp}\left(\mathrm{O}^{t} \mathrm{Bu}\right)$ & Leu & $0.20 \pm 0.035$ \\
\hline $3 \mathbf{c}$ & $\mathrm{Cbz}$ & $\mathrm{Glu}\left(\mathrm{O}^{t} \mathrm{Bu}\right)$ & Phe & $0.028 \pm 0.006$ \\
\hline 3d & $\mathrm{Cbz}$ & $\mathrm{Glu}\left(\mathrm{O}^{t} \mathrm{Bu}\right)$ & Leu & $0.089 \pm 0.02$ \\
\hline $3 e$ & $\mathrm{Cbz}$ & Phe & Leu & $0.85 \pm 0.047$ \\
\hline $3 f$ & $\mathrm{Cbz}$ & $\operatorname{Arg}\left(\mathrm{NO}_{2}\right)$ & Leu & $>50$ \\
\hline $3 g$ & $\mathrm{Cbz}$ & $\operatorname{Arg}(\mathrm{Tos})$ & Leu & $>50$ \\
\hline $3 h$ & $\mathrm{Cbz}$ & $\mathrm{Nap}^{\mathrm{a}}$ & Leu & $0.41 \pm 0.082$ \\
\hline $3 \mathbf{i}$ & Boc & Asp(OBzl) & Phe & $4.83 \pm 2.30$ \\
\hline $3 \mathbf{j}$ & Boc & Asp (OBzl) & Leu & $20.3 \pm 2.05$ \\
\hline $3 \mathbf{k}$ & Boc & Glu(OBzl) & Phe & $7.14 \pm 1.93$ \\
\hline 31 & Boc & Glu(OBzl) & Leu & $>50$ \\
\hline $3 m$ & Boc & Pro & Phe & $>50$ \\
\hline $3 n$ & Boc & Pro & Leu & $>50$ \\
\hline 30 & Boc & Ser(OBzl) & Leu & $0.050 \pm 0.002$ \\
\hline $3 \mathbf{p}$ & Boc & Thr(OBzl) & Leu & $0.29 \pm 0.021$ \\
\hline $\mathbf{3 q}$ & Boc & Tyr(OBzl) & Leu & $>50$ \\
\hline MG132 (3r) & $\mathrm{Cbz}$ & Leu & Leu & $0.28 \pm 0.06$ \\
\hline
\end{tabular}

To fully understand the SAR of inhibitors, we constructed a binding mode of the peptide aldehydes with the $\beta 5$ subunit of the $20 \mathrm{~S}$ proteasome based on the crystal structure of $20 \mathrm{~S}$ proteasome complexed with MG101. Though docking and biochemical data are often not easily comparable, the insights gained into the binding behavior by molecular modeling is meaningful. Given that covalent binding is a unique feature of peptide aldehyde inhibitors, we adopted a covalent docking approach and then developed a protocol to investigate the binding mode of peptide aldehydes with the $20 \mathrm{~S}$ proteasome. The binding mode of the control MG132 is similar to that of MG101 observed in the crystal structure (Figure 2a). MG132 adopts a $\beta$-conformation and fills the gap between strands S2 and S4 by forming hydrogen bonds with residues Thr21, Gly47, and Ala49 and generating an anti-parallel $\beta$-sheet structure (Figure 2b) [10]. The P1-leucine side chain of MG132 projects into the S1 pocket and the $\mathrm{P} 2$-leucine side chain is towards outside. The P3-leucine side chain stretches out into the subunit-specific $\mathrm{S} 3$ pocket and is in close contact with residues of the adjacent $\beta 6$ subunit. 
Other peptide aldehydes are also docked into the 20S proteasome using the same protocol. Similar orientations of $\mathrm{P} 1-\mathrm{P} 4$ residues are found in the docked conformations. For example, the P1-P4 residues of 3c are towards the S1-S4 pockets, respectively, like in MG132 (Figure 2a), and so do the hydrogen bonds (Figure $2 b$ ).

Figure 2. (a) Binding modes of 1 (green), 2 (magenta) and 3c (purple) with $20 \mathrm{~S}$ proteasome. $\beta 5$ and $\beta 6$ subunits are shown in green and yellow, respectively. (b) The binding sites of proteasome and $\mathbf{1}$ (green), $\mathbf{2}$ (magenta) and $\mathbf{3 c}$ (purple). Only backbones and key residues of active sites are shown. (c) Binding modes of 3a which is shown in magenta and $3 \mathbf{c}$ in purple. The side chains at P3 position project into S3 pocket. (d) Binding modes of $\mathbf{3 j}$ (pink) and $\mathbf{3 l}$ (green). The side chains at the P3 position project into S3 pocket. (e) Chymotryptic-like active site in binding modes with $\mathbf{1}$ (green) and $\mathbf{3 m}$ (orange). (f) Key contacts between residues of the ligand binding site of the $20 \mathrm{~S}$ proteasome core particle and the backbones of $\mathbf{1}$ (green) and $\mathbf{3 m}$ (orange).

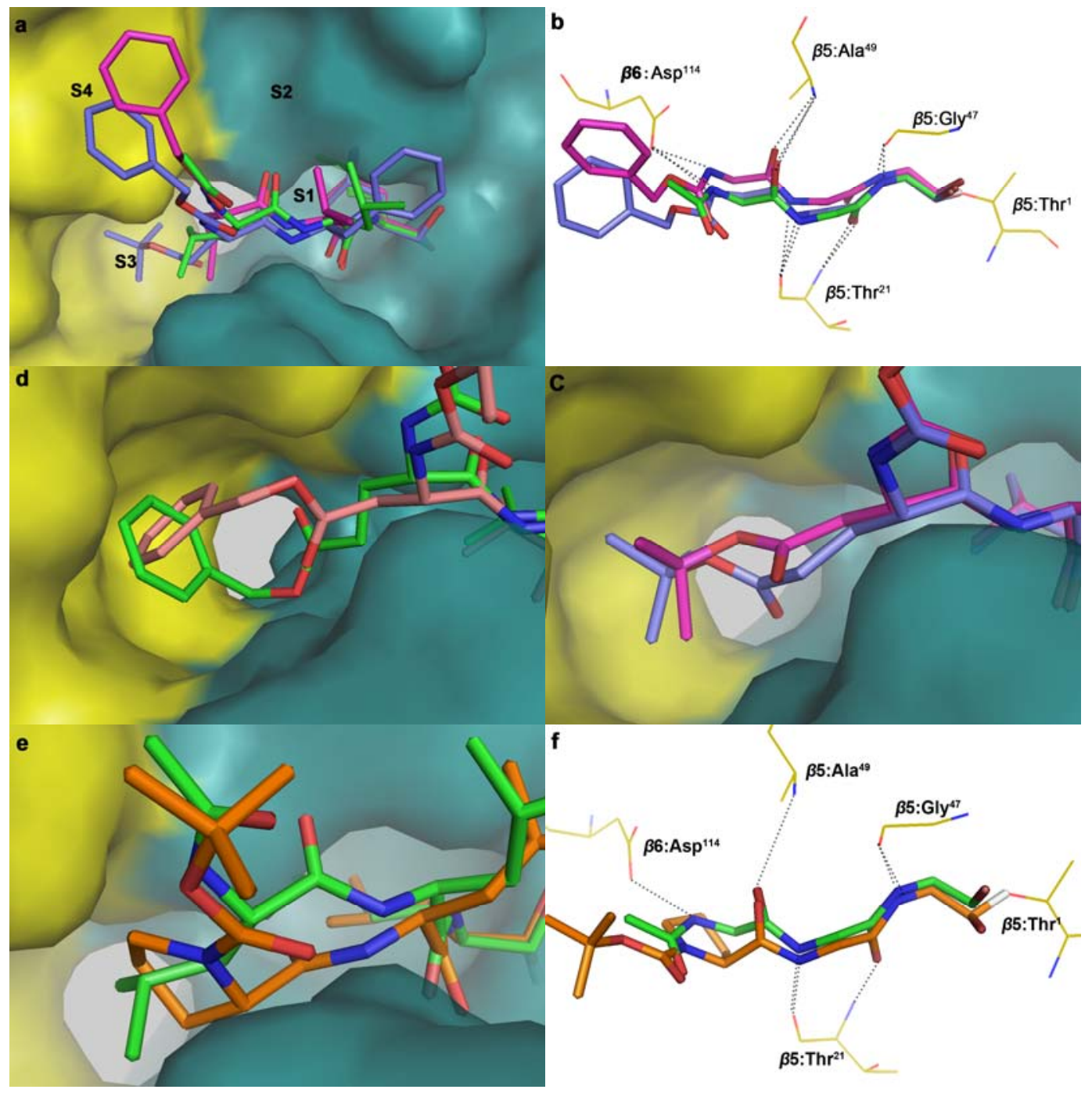

Biochemical investigation shows that the size and length of the P3 side chain is crucial to activity $[42,43]$. Among the $\mathrm{Cbz}$ series compounds, $\mathrm{Glu}\left(\mathrm{O}^{t} \mathrm{Bu}\right)$ residues at $\mathrm{P} 3$ (3c and $\mathbf{3 d}$ ) give the most active inhibition. The structure of the 20S proteasome shows that the $\beta 5$ and $\beta 6$ subunits constitute the 
binding cleft of the S3 pocket, which is able to accommodate long and linear side chains. The docking results show that the tert-butyl glutamic ester (3c) fits this site better than tert-butyl aspartic ester (3a) and provides a strong hydrophobic interaction with the $\beta 5 / \beta 6$ interface (Figure $2 \mathrm{c}$ ). Among the Boc series of compounds, when a phenyl ester was used to replace a tert-butyl ester at P3, the Asp(OBzl) residue (3j) exhibited more active inhibition than $\mathrm{Glu}(\mathrm{OBzl})(\mathbf{3 l})$. According to the docking analysis (Figure $2 \mathrm{~d})$, although the phenyl ester of both $\mathrm{Asp}(\mathrm{OBzl})$ and $\mathrm{Glu}(\mathrm{OBzl})$ project into the S3 pocket, the large sized benzene ring makes the conformation rigid and pushes the backbone slightly out of the original orientation, so the shorter side chain of $\mathrm{Asp}(\mathrm{OBzl})$ is more suitable for the cleft. The most suitable length of side chain in this Boc-series is $\operatorname{Ser}(\mathrm{OBzl})$, and it gives most active inhibition to ChT-L. When the residue at the P3 position is changed to proline (3m and $\mathbf{3 n}$ ), the results show that the pyrrolidine moiety projects into the S3 pocket (Figure 2e), which makes the binding model of the main chain different from that of MG132 (Figure 2f), and resulting in the disappearance of activity.

\section{Experimental}

\subsection{Chemistry}

\subsubsection{General}

Unless specified otherwise, all starting materials and reagents were used as obtained from commercial suppliers without further purification. Thin layer chromatography was performed using silica gel GF-254 (Qing-Dao Chemical Company, China) plated with detection by UV, and column chromatography was performed on silica gel (200-300 mesh, Qing-Dao Chemical Company, China). Optical rotations were recorded on a Perkin-Elmer 243B polarimeter. ${ }^{1} \mathrm{H}-\mathrm{NMR}$ (300 or $500 \mathrm{MHz}$ ) spectra was recorded on Varian VXR-300 and Varian Inova VXR-500 spectrometer. Mass spectra $\left(\right.$ ESI-TOF $\left.^{+} \mathrm{MS}\right)$ was obtained on a MDS SCLEX QSTAR instrument and only the most representative peaks were reported $(\mathrm{m} / \mathrm{z})$.

\subsubsection{Synthesis}

L-Leucinol (5) [39]. Sodium borohydride (1.42 g, $37 \mathrm{mmol}, 2.4$ eq.) was dissolved in anhydrous THF $(40 \mathrm{~mL})$ and L-leucine $(2.00 \mathrm{~g}, 15 \mathrm{mmol}, 1$ eq.) was added in one portion. The solution was cooled to $-5{ }^{\circ} \mathrm{C}$ in an ice-salt bath, and a solution of iodine $(3.87 \mathrm{~g}, 15 \mathrm{mmol}, 1$ eq. $)$ in anhydrous THF $(10 \mathrm{~mL})$ was added dropwise over $40 \mathrm{~min}$. After the gas evolution was ceased, the reaction solution was refluxed for $16 \mathrm{~h}$. The solution was cooled to room temperature and methanol was added cautiously until the mixture became clear. After stirring $30 \mathrm{~min}$, the solution was evaporated and the residue was dissolved by addition of $45 \mathrm{~mL}$ aqueous $\mathrm{NaOH}$. The solution was stirred for $2.5 \mathrm{~h}$ and extracted with methylene chloride $(30 \mathrm{~mL} \times 4)$. The combined organic extracts were dried over $\mathrm{Na}_{2} \mathrm{SO}_{4}$ and concentrated, affording crude product which was distilled under reduced pressure to yield colorless oil $5\left(1.59 \mathrm{~g}, 89.3 \%, 80-83{ }^{\circ} \mathrm{C} / 25 \mathrm{mmHg}\right) \cdot[\alpha]_{\mathrm{D}}^{20}+4.0(\mathrm{c} 0.3, \mathrm{MeOH}) .{ }^{1} \mathrm{H}-\mathrm{NMR}\left(500 \mathrm{MHz}, \mathrm{CDCl}_{3}\right): \delta 0.91$ $(\mathrm{d}, 6 \mathrm{H}, J=6.5 \mathrm{~Hz}), 1.19-1.14(\mathrm{~m}, 2 \mathrm{H}), 1.70-1.65(\mathrm{~m}, 1 \mathrm{H}), 2.04($ br s, 3H, NH, $\mathrm{OH}), 3.23(\mathrm{dd}, 1 \mathrm{H}$, $J=3.0,8.0 \mathrm{~Hz}), 3.57(\mathrm{dd}, 1 \mathrm{H}, J=3.0,8.0 \mathrm{~Hz}) . \mathrm{MS}\left(\mathrm{ESI}_{-} \mathrm{TOF}^{+}\right): 118[\mathrm{M}+\mathrm{H}]^{+}$. 
Boc-L-Phenylalanine-L-Leucinol (6a). Boc-L-phenylalanine (0.25 g, $0.95 \mathrm{mmol}, 1.0$ eq.), L-leucinol (0.20 g, $1.04 \mathrm{mmol}, 1.0$ eq.), and HOBt (0.14 g, $1.0 \mathrm{mmol}, 1.1$ eq.) were mixed in anhydrous THF (2 mL). N,N'-Dicyclohexylcarbodiimide (DCC, $0.22 \mathrm{~g}, 1.0 \mathrm{mmol}, 1.1$ eq.) and 5 (0.11 g, $0.95 \mathrm{mmol}$, 1 eq.) was added at $0{ }^{\circ} \mathrm{C}$, and the mixture was warmed to room temperature and stirred for $16 \mathrm{~h}$. After filtration to remove dicyclohexylurea, the solvent was removed and the residue was partitioned between EtOAc $(20 \mathrm{~mL})$ and $\mathrm{H}_{2} \mathrm{O}(10 \mathrm{~mL})$. The organic phase was washed with $10 \%$ citric acid $(10 \mathrm{~mL} \times 3)$, saturated $\mathrm{NaHCO}_{3}(10 \mathrm{~mL} \times 3)$, and then brine $(10 \mathrm{~mL} \times 2)$. The solution was dried over $\mathrm{Na}_{2} \mathrm{SO}_{4}$ and evaporated to an amorphous solid. The crude product was purified by flash chromatography on silica gel to give compound $\mathbf{6 a}$ as white solid $(0.25 \mathrm{~g}, 71.2 \%)$. $[\alpha]_{\mathrm{D}}^{20}-19.4(\mathrm{c} 0.3$, $\mathrm{MeOH}) .{ }^{1} \mathrm{H}-\mathrm{NMR}\left(500 \mathrm{MHz}, \mathrm{CDCl}_{3}\right): \delta 0.87(\mathrm{~d}, 6 \mathrm{H}, J=7.2 \mathrm{~Hz}), 1.26-1.23(\mathrm{~m}, 2 \mathrm{H}), 1.42(\mathrm{~s}, 9 \mathrm{H})$, 1.50-1.46 (m, 1H), $1.95(\mathrm{~s}, 1 \mathrm{H}), 3.02-2.98(\mathrm{~m}, 1 \mathrm{H}), 3.11-3.07(\mathrm{~m}, 1 \mathrm{H}), 3.35(\mathrm{~m}, 1 \mathrm{H}), 3.48(\mathrm{~d}, 1 \mathrm{H}$, $J=11.0 \mathrm{~Hz}), 3.95-3.93(\mathrm{~m}, 1 \mathrm{H}), 4.25(\mathrm{~m}, 1 \mathrm{H}), 5.06(\mathrm{~s}, 1 \mathrm{H}), 5.60(\mathrm{~d}, 1 \mathrm{H}), 7.28-7.14(\mathrm{~m}, 5 \mathrm{H})$. MS(ESI-TOF $\left.{ }^{+}\right): 345[\mathrm{M}+\mathrm{H}]^{+}$.

Boc-L-Leucine-L-Leucinol (6b). Compound $\mathbf{6 b}$ was obtained by using similar procedure as $\mathbf{6 a}$. White solid. $[\alpha]_{\mathrm{D}}^{20}-11.6(\mathrm{c} 0.52, \mathrm{MeOH}) .{ }^{1} \mathrm{H}-\mathrm{NMR}\left(500 \mathrm{MHz}, \mathrm{CDCl}_{3}\right): \delta 0.93-0.88(\mathrm{~m}, 12 \mathrm{H}), 1.32-1.27(\mathrm{~m}$, $1 \mathrm{H}), 1.40-1.36(\mathrm{~m}, 1 \mathrm{H}), 1.42(\mathrm{~s}, 9 \mathrm{H}), 1.64-1.60(\mathrm{~m}, 4 \mathrm{H}), 2.63(\mathrm{~s}, 1 \mathrm{H}), 3.51-3.46(\mathrm{~m}, 1 \mathrm{H}), 3.69-3.63$ $(\mathrm{m}, 1 \mathrm{H}), 4.01-3.98(\mathrm{~m}, 2 \mathrm{H}), 4.84(\mathrm{~s}, 1 \mathrm{H}), 6.20(\mathrm{~d}, 1 \mathrm{H}, J=8.0 \mathrm{~Hz})$. MS (ESI-TOF $\left.{ }^{+}\right): 331[\mathrm{M}+\mathrm{H}]^{+}$.

TFA L-Phenylalanine-L-Leucinol (7a). To a suspension of $6 \mathbf{a}(0.50 \mathrm{~g}, 1.27 \mathrm{mmol})$ in $\mathrm{CH}_{2} \mathrm{Cl}_{2}(3 \mathrm{~mL})$ was added TFA $(1 \mathrm{~mL})$ at $0{ }^{\circ} \mathrm{C}$. After stirred at room temperature for $2 \mathrm{~h}$, the solution was evaporated and the crude product was used in the next step without purification. TFA L-Leucine-L-Leucinol (7b) was prepared by a similar procedure.

Cbz-L-Asp(OtBu)-L-Phe-L-Leuninol (8a). Cbz-L-Asp(OtBu)-OH (0.20 g, $0.62 \mathrm{mmol}$, 1 eq.) was dissolved in anhydrous THF ( $5 \mathrm{~mL})$, HOBt $(92 \mathrm{mg}, 0.68 \mathrm{mmol}, 1.1$ eq.) and DCC (0.14 g, $0.68 \mathrm{mmol}$, 1.1 eq.) were added after cooling to $0{ }^{\circ} \mathrm{C}$. The mixture was stirred for $60 \mathrm{~min}$ then $7 \mathrm{a}(0.21 \mathrm{~g}$, $0.62 \mathrm{mmol}, 1$ eq. $)$ and diisopropylethylamine $(0.22 \mathrm{~mL}, 1.24 \mathrm{mmol}, 2$ eq. $)$ were added. The reaction mixture was stirred at room temperature overnight. The insoluble material was filtered off and the solution was washed successively with $10 \%$ citric acid, saturated $\mathrm{NaHCO}_{3}$ and brine. After drying with $\mathrm{Na}_{2} \mathrm{SO}_{4}$ the solvents were removed under reduced pressure. The crude products were used in the next step without further purification. Compounds $\mathbf{8 b}-\mathbf{r}$ were prepared using similar procedures.

Cbz-L-Asp(OtBu)-L-Phe-L-Leucinal (3a) A solution of oxalyl chloride (63 mg, $0.49 \mathrm{mmol}, 1.4$ eq.) in $\mathrm{CH}_{2} \mathrm{Cl}_{2}(0.4 \mathrm{~mL})$ was cooled to $-78{ }^{\circ} \mathrm{C}$, DMSO (45 $\mu \mathrm{L}, 0.63 \mathrm{mmol}, 1.8$ eq. $)$ in $\mathrm{CH}_{2} \mathrm{Cl}_{2}(0.1 \mathrm{~mL})$ was added dropwise over $10 \mathrm{~min}$. The resulting mixture was stirred for $30 \mathrm{~min}$ at $-78{ }^{\circ} \mathrm{C}$. A solution of $8 \mathbf{a}$ ( $0.20 \mathrm{~g}, 0.35 \mathrm{mmol}, 1$ eq.) in $\mathrm{CH}_{2} \mathrm{Cl}_{2}(1.5 \mathrm{~mL})$ was then added dropwise over $30 \mathrm{~min}$ affording a cloudy mixture which was allowed to stir at $-78{ }^{\circ} \mathrm{C}$ for $2 \mathrm{~h}$. Triethylamine $(0.19 \mathrm{~mL}, 1.41 \mathrm{mmol}, 4$ eq. $)$ was then slowly added over $30 \mathrm{~min}$, and the solution was gradually warmed to room temperature. The reaction mixture was washed with saturated aq $\mathrm{NaHCO}_{3}$, and brine. The organic phase was dried, filtered and concentrated. The crude product was purified by flash chromatography on silica gel to give compound 3a as white solid $(0.10 \mathrm{~g}, 52.3 \%)$. White solid. $[\alpha]_{\mathrm{D}}^{20}-19.1$ (c $\left.0.4, \mathrm{CHCl}_{3}\right)$. ${ }^{1} \mathrm{H}-\mathrm{NMR}$ 
(300 MHz, $\left.\mathrm{CDCl}_{3}\right): \delta 0.86(\mathrm{~d}, 6 \mathrm{H}, J=6.2 \mathrm{~Hz}), 1.40(\mathrm{~s}, 9 \mathrm{H}), 1.70-1.53(\mathrm{~m}, 3 \mathrm{H}), 2.73-2.68(\mathrm{~m}, 2 \mathrm{H})$, 3.10-3.04 (m, 2H), 4.38-4.30 (m, 2H), $4.71(\mathrm{~d}, 1 \mathrm{H}, J=7.2 \mathrm{~Hz}), 5.09(\mathrm{~d}, 2 \mathrm{H}, J=3.6 \mathrm{~Hz}), 5.72(\mathrm{~m}$, $1 \mathrm{H}), 6.58(\mathrm{~d}, 1 \mathrm{H}, J=7.5 \mathrm{~Hz}), 6.82(\mathrm{t}, 1 \mathrm{H}, J=8.7 \mathrm{~Hz}), 7.38-7.19(\mathrm{~m}, 10 \mathrm{H}), 9.43(\mathrm{~d}, 1 \mathrm{H}) . \mathrm{MS}$ $\left(\right.$ ESI-TOF ${ }^{+}$): $568[\mathrm{M}+\mathrm{H}]^{+}$. Elemental Anal.Calcd. for $\mathrm{C}_{31} \mathrm{H}_{41} \mathrm{~N}_{3} \mathrm{O}_{7}: \mathrm{C}, 65.59 ; \mathrm{H}, 7.28 ; \mathrm{N}, 7.40$. found: C, 65.67; H, 7.48; N, 7.32.

Compounds 3b-3r were obtained using a similar procedure as $\mathbf{3 a}$.

Cbz-L-Asp $(\mathrm{OtBu})$-L-Leu-L-Leucinal (3b): White solid. [ $\alpha]_{\mathrm{D}}^{20}-21.0\left(\mathrm{c} 0.5, \mathrm{CHCl}_{3}\right) .{ }^{1} \mathrm{H}-\mathrm{NMR}(300 \mathrm{MHz}$, $\left.\mathrm{CDCl}_{3}\right): \delta 0.97-0.88(\mathrm{~m}, 12 \mathrm{H}), 1.42(\mathrm{~s}, 9 \mathrm{H}), 1.69-1.57(\mathrm{~m}, 6 \mathrm{H}), 4.48-4.43(\mathrm{~m}, 3 \mathrm{H}), 5.13(\mathrm{~d}, 2 \mathrm{H}$, $J=3.6 \mathrm{~Hz}), 5.88(\mathrm{~s}, 1 \mathrm{H}), 6.67-6.65(\mathrm{~m}, 2 \mathrm{H}), 7.37(\mathrm{~m}, 5 \mathrm{H}), 9.52(\mathrm{~s}, 1 \mathrm{H}) . \mathrm{MS}\left(\mathrm{ESI}_{-} \mathrm{TOF}^{+}\right): 534[\mathrm{M}+\mathrm{H}]^{+}$. Elemental Anal.Calcd. for $\mathrm{C}_{28} \mathrm{H}_{43} \mathrm{~N}_{3} \mathrm{O}_{7}$ : C, 63.02; H, 8.12; N, 7.87. found: C, 63.09; H, 8.19; N, 7.88.

Cbz-L-Glu(OtBu)-L-Phe-L-Leucinal (3c): White solid. $[\alpha]_{\mathrm{D}}^{20}-28.1$ (c 0.6, CHCl3). ${ }^{1} \mathrm{H}-\mathrm{NMR}(300 \mathrm{MHz}$, $\left.\mathrm{CDCl}_{3}\right): \delta 0.90(\mathrm{~d}, 6 \mathrm{H}, J=5.4 \mathrm{~Hz}), 1.44(\mathrm{~s}, 9 \mathrm{H}), 1.56-1.49(\mathrm{~m}, 3 \mathrm{H}), 1.91-1.88(\mathrm{~m}, 2 \mathrm{H}), 2.25(\mathrm{t}, 2 \mathrm{H}$, $J=6.3 \mathrm{~Hz}), 3.14(\mathrm{t}, 2 \mathrm{H}, J=8.4 \mathrm{~Hz}), 4.06-3.96(\mathrm{~m}, 1 \mathrm{H}), 4.38-4.33(\mathrm{~m}, 1 \mathrm{H}), 4.75-4.69(\mathrm{~m}, 1 \mathrm{H}), 5.04$ $(\mathrm{dd}, 2 \mathrm{H}, J=8.3,22.2 \mathrm{~Hz}), 6.02(\mathrm{~s}, 1 \mathrm{H}), 6.70-6.64(\mathrm{~m}, 1 \mathrm{H}), 7.35-7.18(\mathrm{~m}, 10 \mathrm{H}), 9.43(\mathrm{~d}, 1 \mathrm{H}, J=4.8$ Hz). MS (ESI-TOF ${ }^{+}$): $582[\mathrm{M}+\mathrm{H}]^{+}$. Elemental Anal.Calcd. for $\mathrm{C}_{32} \mathrm{H}_{43} \mathrm{~N}_{3} \mathrm{O}_{7}$ : C, 66.07; H, 7.45; N, 7.22. found: C, 65.99; $\mathrm{H}, 7.45 ; \mathrm{N}, 7.26$.

Cbz-L-Glu(OtBu)-L-leu-L-Leucinal (3d): White solid. $[\alpha]_{\mathrm{D}}^{20}-14.4\left(\right.$ c $\left.0.7, \mathrm{CHCl}_{3}\right) .{ }^{1} \mathrm{H}-\mathrm{NMR}(300 \mathrm{MHz}$, $\left.\mathrm{CDCl}_{3}\right): \delta 0.97-0.90(\mathrm{~m}, 6 \mathrm{H}), 1.44(\mathrm{~s}, 9 \mathrm{H}), 1.74-1.54(\mathrm{~m}, 6 \mathrm{H}), 2.09-1.96(\mathrm{~m}, 2 \mathrm{H}), 2.40(\mathrm{t}, 2 \mathrm{H}, J=5.1$ $\mathrm{Hz}), 4.18(\mathrm{t}, 1 \mathrm{H}, J=7.2 \mathrm{~Hz}), 4.49-4.38(\mathrm{~m}, 2 \mathrm{H}), 5.10$ (d, 2H, $J=3.3 \mathrm{~Hz}), 6.14(\mathrm{~s}, 1 \mathrm{H}), 6.62(\mathrm{~s}, 1 \mathrm{H})$, $6.96(\mathrm{~s}, 1 \mathrm{H}), 7.35-7.26(\mathrm{~m}, 5 \mathrm{H}), 9.52(\mathrm{~d}, 1 \mathrm{H}, J=9.3 \mathrm{~Hz}) . \mathrm{MS}\left(\mathrm{ESI}_{-} \mathrm{TOF}^{+}\right): 548[\mathrm{M}+\mathrm{H}]^{+}$. Elemental Anal.Calcd. for $\mathrm{C}_{29} \mathrm{H}_{45} \mathrm{~N}_{3} \mathrm{O}_{7}$ : C, 63.60; H, 8.28; N, 7.67. found: C, 62.54; H, 8.19; N, 7.70.

Cbz-L-Phe-L-leu-L-Leucinal (3e): White solid, $[\alpha]_{\mathrm{D}}^{20}-16.1$ (c $\left.0.3, \mathrm{CHCl}_{3}\right) .{ }^{1} \mathrm{H}-\mathrm{NMR}(500 \mathrm{MHz}$, $\left.\mathrm{CDCl}_{3}\right): \delta 0.92(\mathrm{~m}, 12 \mathrm{H}), 1.74-1.32(\mathrm{~m}, 6 \mathrm{H}), 4.36-4.33(\mathrm{~m}, 1 \mathrm{H}), 4.54-4.47(\mathrm{~m}, 2 \mathrm{H}), 5.04-5.00(\mathrm{~d}$, $2 \mathrm{H}, J=12.5 \mathrm{~Hz}), 5.45-5.40(\mathrm{~m}, 1 \mathrm{H}), 7.00-6.53(\mathrm{~m}, 2 \mathrm{H}), 7.36-7.16(\mathrm{~m}, 10 \mathrm{H}), 9.50(\mathrm{~s}, 1 \mathrm{H}) . \mathrm{MS}$ $\left(\right.$ ESI-TOF ${ }^{+}$): $544[\mathrm{M}+\mathrm{H}]^{+}$. Elemental Anal.Calcd. for $\mathrm{C}_{32} \mathrm{H}_{37} \mathrm{~N}_{3} \mathrm{O}_{5}: \mathrm{C}, 70.70 ; \mathrm{H}, 6.86 ; \mathrm{N}, 7.73$. Found: C, 70.74; H, 6.90; N, 7.65.

Cbz-L-Arg $\left(\mathrm{NO}_{2}\right)$-L-leu-L-Leucinal (3f): White solid, $[\alpha]_{\mathrm{D}}^{20}-67.2$ (c 0.1, $\left.\mathrm{CHCl}_{3}\right) .{ }^{1} \mathrm{H}-\mathrm{NMR}(500 \mathrm{MHz}$, $\left.\mathrm{CDCl}_{3}\right): \delta 0.88-0.86(\mathrm{~m}, 12 \mathrm{H}), 1.66-1.38(\mathrm{~m}, 8 \mathrm{H}), 1.80-1.75(\mathrm{~m}, 2 \mathrm{H}), 3.28-3.24(\mathrm{~m}, 2 \mathrm{H}), 4.07$ (dd, $1 \mathrm{H}, J=4.5,15.5 \mathrm{~Hz}), 4.20-4.16(\mathrm{~m}, 1 \mathrm{H}), 4.39-4.30(\mathrm{~m}, 2 \mathrm{H}), 5.08(\mathrm{~s}, 2 \mathrm{H}), 5.98(\mathrm{~s}, 1 \mathrm{H}), 6.49-6.33(\mathrm{~m}$, 1H), 7.35-7.30 (m, 6H), $7.47(\mathrm{~s}, 1 \mathrm{H}), 8.45(\mathrm{~s}, 1 \mathrm{H}), 9.52(\mathrm{~s}, 1 \mathrm{H})$. MS (ESI-TOF $\left.{ }^{+}\right): 564[\mathrm{M}+\mathrm{H}]^{+}$. Elemental Anal.Calcd. for $\mathrm{C}_{26} \mathrm{H}_{41} \mathrm{~N}_{7} \mathrm{O}_{7}, \mathrm{C}, 55.40 ; \mathrm{H}, 7.33 ; \mathrm{N}, 17.40$. Found: C, 55.32; H, 7.42; N, 17.30 .

Cbz-L-Arg(Tos)-L-leu-L-Leucinal (3g): White solid. [ $\alpha]_{\mathrm{D}}^{20}-12.3$ (c 0.2, $\left.\mathrm{CHCl}_{3}\right) .{ }^{1} \mathrm{H}-\mathrm{NMR}(500 \mathrm{MHz}$, $\left.\mathrm{CDCl}_{3}\right): \delta 1.11-0.86(\mathrm{~m}, 12 \mathrm{H}), 1.74-1.31(\mathrm{~m}, 8 \mathrm{H}), 1.95-1.92(\mathrm{~m}, 2 \mathrm{H}), 2.42(\mathrm{~s}, 3 \mathrm{H}), 2.49-2.47(\mathrm{~m}$, 2H), 4.20-4.15 (m, 1H), 4.32-4.26 (m, 2H), 5.15 (s, 2H), 5.98 (s, 1H), 6.49-6.43 (m, 1H), 7.38-7.25 
(m, 6H), $7.81(\mathrm{~d}, 1 \mathrm{H}), 8.11(\mathrm{~s}, 1 \mathrm{H}), 9.47(\mathrm{~s}, 1 \mathrm{H}) . \mathrm{MS}_{\left(\mathrm{ESI}_{-} \mathrm{TOF}^{+}\right):} 673[\mathrm{M}+\mathrm{H}]^{+}$. Elemental Anal.Calcd. for $\mathrm{C}_{33} \mathrm{H}_{48} \mathrm{~N}_{6} \mathrm{O}_{7} \mathrm{~S}, \mathrm{C}, 58.91 ; \mathrm{H}, 7.19 ; \mathrm{N}, 12.49$. found: $\mathrm{C}, 58.99 ; \mathrm{H}, 7.12 ; \mathrm{N}, 12.36$.

Cbz-L-(2-Naphthyl)alanine-L-Leu-L-Leucinal (3h): White solid. $[\alpha]_{\mathrm{D}}^{20}-51.1$ (c $\left.0.7, \mathrm{CHCl}_{3}\right) .{ }^{1} \mathrm{H}-\mathrm{NMR}$ $\left(500 \mathrm{MHz}, \mathrm{CDCl}_{3}\right): \delta 0.93-0.89(\mathrm{~m}, 12 \mathrm{H}), 1.91-1.46(\mathrm{~m}, 6 \mathrm{H}), 3.22-3.16(\mathrm{dd}, 2 \mathrm{H}, J=3.9,7.5 \mathrm{~Hz})$, $4.48-4.43(\mathrm{~m}, \mathrm{H}), 4.89-4.75(\mathrm{~m}, 2 \mathrm{H}), 5.12(\mathrm{~d}, 2 \mathrm{H}, J=4.2 \mathrm{~Hz}), 6.03(\mathrm{~d}, 1 \mathrm{H}), 6.69-6.58(\mathrm{~m}, 2 \mathrm{H})$, $7.38-7.31(\mathrm{~m}, 6 \mathrm{H}), 7.51(\mathrm{t}, 1 \mathrm{H}, J=7.5 \mathrm{~Hz}), 7.58-7.55(\mathrm{~m}, 1 \mathrm{H}), 7.77(\mathrm{~d}, 1 \mathrm{H}, J=8 \mathrm{~Hz}), 7.88(\mathrm{~d}, 1 \mathrm{H}$, $J=8.0 \mathrm{~Hz}), 8.26(\mathrm{~d}, 1 \mathrm{H}, J=9.0 \mathrm{~Hz}), 9.51(\mathrm{~d}, 1 \mathrm{H}, J=6.0 \mathrm{~Hz})$. MS (ESI-TOF $\left.{ }^{+}\right): 560[\mathrm{M}+\mathrm{H}]^{+}$. Elemental Anal.Calcd. for $\mathrm{C}_{33} \mathrm{H}_{41} \mathrm{~N}_{3} \mathrm{O}_{5}$ : C, 70.82; H, 7.38; N, 7.51. found: C, 70.90; H, 7.42; N, 7.61.

Boc-L-Asp(OBzl)-L-Phe-L-Leucinal (3i): White solid. $[\alpha]_{\mathrm{D}}^{20}-19.5$ (c $\left.0.65, \mathrm{CHCl}_{3}\right) .{ }^{1} \mathrm{H}-\mathrm{NMR}(300 \mathrm{MHz}$, $\left.\mathrm{CDCl}_{3}\right): \delta 0.94-0.88(\mathrm{~m}, 6 \mathrm{H}), 1.40(\mathrm{~s}, 9 \mathrm{H}), 1.60-1.54(\mathrm{~m}, 1 \mathrm{H}), 1.72-1.66(\mathrm{~m}, 2 \mathrm{H}), 3.04-2.77(\mathrm{~m}, 3 \mathrm{H})$, 3.26-3.23 (m, 1H), 4.18-4.10 (m, 2H), 4.71-4.67 (m, 1H), 5.11-5.04 (m, 2H), $5.44(\mathrm{~m}, 1 \mathrm{H}), 6.59$ $(\mathrm{s}, 1 \mathrm{H}), 6.80(\mathrm{~s}, 1 \mathrm{H}), 7.35-7.21(\mathrm{~m}, 10 \mathrm{H}), 9.40(\mathrm{~s}, 1 \mathrm{H}) . \mathrm{MS}\left(\mathrm{ESI}_{-T O F}{ }^{+}\right): 568[\mathrm{M}+\mathrm{H}]^{+}$. Elemental Anal.Calcd. for $\mathrm{C}_{31} \mathrm{H}_{41} \mathrm{~N}_{3} \mathrm{O}_{7}$ : C, 65.59; H, 7.28; N, 7.40. Found: C, 65.38; H, 7.19; N, 7.47.

Boc-L-Asp $\left(\mathrm{OBz}\right.$ )-L-Leu-L-Leucinal (3j): White solid. $[\alpha]_{\mathrm{D}}^{20}-44.5$ (c $\left.0.7, \mathrm{CHCl}_{3}\right) .{ }^{1} \mathrm{H}-\mathrm{NMR}(300 \mathrm{MHz}$, $\left.\mathrm{CDCl}_{3}\right): \delta$ 0.95-0.92 (m, 12H), $1.44(\mathrm{~s}, 9 \mathrm{H}), 1.70-1.59(\mathrm{~m}, 6 \mathrm{H}), 2.92(\mathrm{t}, 2 \mathrm{H}, J=5.7 \mathrm{~Hz}), 4.47-4.40$ $(\mathrm{m}, 3 \mathrm{H}), 5.22(\mathrm{~s}, 2 \mathrm{H}), 5.48(\mathrm{~d}, 1 \mathrm{H}), 6.70(\mathrm{~d}, 1 \mathrm{H}, J=6.9 \mathrm{~Hz}), 6.87(\mathrm{~m}, 1 \mathrm{H}), 7.40-7.36(\mathrm{~m}, 5 \mathrm{H}), 9.50$ (s, 1H). MS (ESI-TOF ${ }^{+}$): $534[\mathrm{M}+\mathrm{H}]^{+}$. Elemental Anal.Calcd. for $\mathrm{C}_{28} \mathrm{H}_{43} \mathrm{~N}_{3} \mathrm{O}_{7}: \mathrm{C}, 63.02 ; \mathrm{H}, 8.12 ; \mathrm{N}$, 7.87. found: $\mathrm{C}, 62.65 ; \mathrm{H}, 7.98 ; \mathrm{N}, 7.95$.

Boc-L-Glu(OBzl)-L-Phe-L-Leucinal (3k): White solid. $[\alpha]_{\mathrm{D}}^{20}-18.1\left(\mathrm{c} 0.7, \mathrm{CHCl}_{3}\right) .{ }^{1} \mathrm{H}-\mathrm{NMR}(300 \mathrm{MHz}$, $\left.\mathrm{CDCl}_{3}\right): \delta 0.90-0.87(\mathrm{~m}, 6 \mathrm{H}), 1.37(\mathrm{~s}, 9 \mathrm{H}), 1.69-1.54(\mathrm{~m}, 3 \mathrm{H}), 2.07-2.03(\mathrm{~m}, 2 \mathrm{H}), 2.44(\mathrm{t}, 2 \mathrm{H}, J=4.2$ $\mathrm{Hz}), 3.21(\mathrm{dd}, 2 \mathrm{H}, J=7.2,14.1 \mathrm{~Hz}), 4.05-4.01(\mathrm{~m}, 1 \mathrm{H}), 4.38-4.29(\mathrm{~m}, 1 \mathrm{H}), 4.77-4.74(\mathrm{~m}, 1 \mathrm{H}), 5.13$ $(\mathrm{d}, 2 \mathrm{H}, J=3.3 \mathrm{~Hz}), 5.57(\mathrm{~s}, 1 \mathrm{H}), 6.68-6.56(\mathrm{~m}, 2 \mathrm{H}), 7.38-7.20(\mathrm{~m}, 10 \mathrm{H}), 9.41(\mathrm{~d}, 1 \mathrm{H}, J=5.4 \mathrm{~Hz}) . \mathrm{MS}$ $\left(\right.$ ESI-TOF ${ }^{+}$); $582[\mathrm{M}+\mathrm{H}]^{+}$. Elemental Anal.Calcd. for $\mathrm{C}_{32} \mathrm{H}_{43} \mathrm{~N}_{3} \mathrm{O}_{7}: \mathrm{C}, 66.07 ; \mathrm{H}, 7.45 ; \mathrm{N}, 7.22$. found: C, 65.91; H, 7.56; N, 7.29.

Boc-L-Glu(OBzl)-L-Leu-L-Leucinal (3I): White solid. [ $\alpha]_{\mathrm{D}}^{20}-31.5$ (c $\left.0.8, \mathrm{CHCl}_{3}\right) .{ }^{1} \mathrm{H}-\mathrm{NMR}(300 \mathrm{MHz}$, $\left.\mathrm{CDCl}_{3}\right): \delta 0.95-0.90(\mathrm{~m}, 12 \mathrm{H}), 1.43(\mathrm{~s}, 9 \mathrm{H}), 1.97-1.62(\mathrm{~m}, 6 \mathrm{H}), 2.17-2.12(\mathrm{~m}, 2 \mathrm{H}), 2.57-2.48(\mathrm{~m}, 2 \mathrm{H})$, $4.11(\mathrm{t}, 1 \mathrm{H}, J=5.7 \mathrm{~Hz}), 4.48-4.43(\mathrm{~m}, 2 \mathrm{H}), 5.13(\mathrm{~s}, 2 \mathrm{H}), 5.49(\mathrm{~d}, 1 \mathrm{H}), 6.83(\mathrm{~s}, 1 \mathrm{H}), 7.37-7.27$ $(\mathrm{m}, 5 \mathrm{H}), 9.52(\mathrm{~s}, 1 \mathrm{H})$. MS $\left(\mathrm{ESI}_{-} \mathrm{TOF}^{+}\right): 548[\mathrm{M}+\mathrm{H}]^{+}$. Elemental Anal.Calcd. for $\mathrm{C}_{29} \mathrm{H}_{45} \mathrm{~N}_{3} \mathrm{O}_{7}$ : C, 63.60; H, 8.28; N, 7.67. found: C, 63.71; H, 8.19; N, 7.68.

Boc-L-Pro-L-Phe-L-Leucinal (3m): White solid. $[\alpha]_{\mathrm{D}}^{20}-10.0$ (c $\left.0.5, \mathrm{CHCl}_{3}\right) .{ }^{1} \mathrm{H}-\mathrm{NMR}(300 \mathrm{MHz}$, $\left.\mathrm{CDCl}_{3}\right): \delta 0.98-0.88(\mathrm{~m}, 6 \mathrm{H}), 1.43(\mathrm{~s}, 9 \mathrm{H}), 2.08-1.46(\mathrm{~m}, 7 \mathrm{H}), 3.17-3.13(\mathrm{~m}, 2 \mathrm{H}), 3.40-3.35(\mathrm{~m}, 2 \mathrm{H})$, 4.45-4.38 (m, 2H), 5.00-4.94 (m, 1H), $5.92(\mathrm{~s}, 1 \mathrm{H}), 6.72(\mathrm{~s}, 1 \mathrm{H}),, 7.40-7.20(\mathrm{~m}, 5 \mathrm{H}), 9.45(\mathrm{~s}, 1 \mathrm{H})$. MS $\left(\right.$ ESI-TOF ${ }^{+}$): $460[\mathrm{M}+\mathrm{H}]^{+}$. Elemental Anal.Calcd. for $\mathrm{C}_{25} \mathrm{H}_{37} \mathrm{~N}_{3} \mathrm{O}_{5}: \mathrm{C}, 65.34 ; \mathrm{H}, 8.11 ; \mathrm{N}, 9.14$. found: C, 65.44; H, 8.19; N, 9.15. 
Boc-L-Pro-L-Leu-L-Leucinal (3n): White solid. $[\alpha]_{\mathrm{D}}^{20}-23.3$ (c $0.6, \mathrm{CHCl}_{3}$ ). ${ }^{1} \mathrm{H}-\mathrm{NMR}(300 \mathrm{MHz}$, $\left.\mathrm{CDCl}_{3}\right): \delta 1.01-0.93(\mathrm{~m}, 12 \mathrm{H}), 1.50-1.32(\mathrm{~m}, 14 \mathrm{H}), 1.75-1.68(\mathrm{~m}, 6 \mathrm{H}), 4.16-4.12(\mathrm{~m}, 1 \mathrm{H}), 4.52-4.45$ $(\mathrm{m}, 1 \mathrm{H}), 4.91(\mathrm{~m}, 1 \mathrm{H}), 6.70(\mathrm{~s}, 1 \mathrm{H}), 9.56(\mathrm{~d}, 1 \mathrm{H}, J=2.7 \mathrm{~Hz}) . \mathrm{MS}\left(\mathrm{ESI}_{-} \mathrm{TOF}^{+}\right): 426[\mathrm{M}+\mathrm{H}]^{+}$. Elemental Anal.Calcd. for $\mathrm{C}_{22} \mathrm{H}_{39} \mathrm{~N}_{3} \mathrm{O}_{5}$ : C, 62.09; H, 9.24; N, 9.87. found: C, 61.18; H, 9.28; N, 9.80.

Boc-L-Ser(OBn)-L-Leu-L-Leucinal (3o): White solid. $[\alpha]_{\mathrm{D}}^{20}-35.1$ (c 0.4, $\left.\mathrm{CHCl}_{3}\right) .{ }^{1} \mathrm{H}-\mathrm{NMR}(500 \mathrm{MHz}$, $\left.\mathrm{CDCl}_{3}\right): \delta 0.92-0.88(\mathrm{~m}, 12 \mathrm{H}), 1.80-1.40(\mathrm{~m}, 15 \mathrm{H}), 3.65-3.59(\mathrm{~m}, 1 \mathrm{H}), 3.91-3.86(\mathrm{~m}, 1 \mathrm{H}), 4.52-4.20$ $(\mathrm{m}, 3 \mathrm{H}), 4.54(\mathrm{~s}, 2 \mathrm{H}), 5.39(\mathrm{~s}, 1 \mathrm{H}), 6.94-6.52(\mathrm{~m}, 2 \mathrm{H}), 7.38-7.25(\mathrm{~m}, 5 \mathrm{H}), 9.52$ (brs, 1H). MS $\left(\right.$ ESI-TOF ${ }^{+}$): $506[\mathrm{M}+\mathrm{H}]^{+}$. Elemental Anal.Calcd. for $\mathrm{C}_{27} \mathrm{H}_{43} \mathrm{~N}_{3} \mathrm{O}_{6}: \mathrm{C}, 64.13 ; \mathrm{H}, 8.57 ; \mathrm{N}, 8.31$. found: C, 64.10; H, 8.51; N, 8.40.

Boc-L-Thr(OBn)-L-Leu-L-Leucinal (3p): White solid. [ $\alpha]_{\mathrm{D}}^{20}-6.0\left(\mathrm{c} 0.6, \mathrm{CHCl}_{3}\right) .{ }^{1} \mathrm{H}-\mathrm{NMR}(500 \mathrm{MHz}$, $\left.\mathrm{CDCl}_{3}\right): \delta 0.87-0.84(\mathrm{~m}, 6 \mathrm{H}), 0.99-0.91(\mathrm{~m}, 6 \mathrm{H}), 1.22-1.16(\mathrm{~m}, 3 \mathrm{H}), 1.45(\mathrm{~s}, 9 \mathrm{H}), 1.77-1.50(\mathrm{~m}, 6 \mathrm{H})$, $4.19-4.16(\mathrm{~m}, 1 \mathrm{H}), 4.24-4.20(\mathrm{~m}, 1 \mathrm{H}), 4.36-4.27(\mathrm{~m}, 1 \mathrm{H}), 4.51-4.48(\mathrm{~m}, 1 \mathrm{H}), 4.65(\mathrm{dd}, 1 \mathrm{H}, J=4$, $12.0 \mathrm{~Hz}), 5.45(\mathrm{~s}, 1 \mathrm{H}), 6.87-6.54(\mathrm{~m}, 2 \mathrm{H}), 7.35-7.25(\mathrm{~m}, 5 \mathrm{H}), 9.49(\mathrm{~d}, 1 \mathrm{H}, J=25.0 \mathrm{~Hz}) . \mathrm{MS}$ $\left(\right.$ ESI-TOF ${ }^{+}$): $520[\mathrm{M}+\mathrm{H}]^{+}$. Elemental Anal.Calcd. for $\mathrm{C}_{28} \mathrm{H}_{45} \mathrm{~N}_{3} \mathrm{O}_{6}: \mathrm{C}, 64.71 ; \mathrm{H}, 8.73 ; \mathrm{N}, 8.09$. found: C, 64.78; H, 8.80; N, 8.01.

Boc-L-Tyr $(\mathrm{OBn})$-L-Leu-L-Leucinal (3q): White solid. $[\alpha]_{\mathrm{D}}^{20}-4.6\left(\right.$ c $\left.0.3, \mathrm{CHCl}_{3}\right) .{ }^{1} \mathrm{H}-\mathrm{NMR}(500 \mathrm{MHz}$, $\left.\mathrm{CDCl}_{3}\right): \delta 0.93(\mathrm{~m}, 12), 1.40(\mathrm{~s}, 9 \mathrm{H}), 1.76-1.45(\mathrm{~m}, 6 \mathrm{H}), 3.03-3.00(\mathrm{~m}, 2 \mathrm{H}), 4.29-4.22(\mathrm{~m}, 1 \mathrm{H})$, 4.46-4.40 (m, 1H), $4.89(\mathrm{~d}, 1 \mathrm{H}, J=4.0 \mathrm{~Hz}), 5.04(\mathrm{~s}, 2 \mathrm{H}), 6.28(\mathrm{~d}, 1 \mathrm{H}, J=8.5 \mathrm{~Hz}, \mathrm{NH}), 6.36(\mathrm{~m}, 1 \mathrm{H})$, $6.74(\mathrm{~s}, 1 \mathrm{H}), 6.92(\mathrm{dd}, 2 \mathrm{H}, J=6.0,11.5 \mathrm{~Hz}), 7.11(\mathrm{~d}, 2 \mathrm{H}, J=8 \mathrm{~Hz}), 7.43-7.26(\mathrm{~m}, 5 \mathrm{H}), 9.51(\mathrm{~s}, 1 \mathrm{H})$. MS (ESI-TOF ${ }^{+}$): $582[\mathrm{M}+\mathrm{H}]^{+}$. Elemental Anal.Calcd. for $\mathrm{C}_{33} \mathrm{H}_{47} \mathrm{~N}_{3} \mathrm{O}_{6}: \mathrm{C}, 68.13 ; \mathrm{H}, 8.14 ; \mathrm{N}, 7.22$. found: C, 68.07; H, 8.19; N, 7.16.

Cbz-L-Leu-L-Leu-L-Leucinal (MG132, 3r): White solid. $[\alpha]_{\mathrm{D}}^{20}-14.4$ (c $0.8, \mathrm{CHCl}_{3}$ ). ${ }^{1} \mathrm{H}-\mathrm{NMR}(300$ $\left.\mathrm{MHz}, \mathrm{CDCl}_{3}\right): \delta 0.93-0.88(\mathrm{~m}, 18 \mathrm{H}), 1.65-1.58(\mathrm{~m}, 9 \mathrm{H}), 4.18-4.14(\mathrm{t}, 1 \mathrm{H}, J=5.5 \mathrm{~Hz}), 4.47-4.40(\mathrm{~m}$, 2H), 5.09-4.89 (m, 2H), $5.44(\mathrm{~d}, 1 \mathrm{H}, J=22.5 \mathrm{~Hz}), 6.66(\mathrm{~d}, 1 \mathrm{H}, J=8.0 \mathrm{~Hz}), 6.95$ (d, 1H, $J=33.5 \mathrm{~Hz})$, $7.28-7.38(\mathrm{~m}, 5 \mathrm{H}), 9.52(\mathrm{~d}, 1 \mathrm{H}) . \mathrm{MS}\left(\mathrm{ESI}_{-} \mathrm{TOF}^{+}\right): 476[\mathrm{M}+\mathrm{H}]^{+}$. Elemental Anal.Calcd. for $\mathrm{C}_{26} \mathrm{H}_{41} \mathrm{~N}_{3} \mathrm{O}_{5}$ : C, 65.66; H, 8.69; N, 8.83. found: C, 65.70; H, 8.62; N, 8.79.

\subsection{Biological Testing}

Assays for proteasome activities. The enzymatic activities of the proteasome were assayed using fluorogenic peptides: Suc-Leu-Leu-Val-Tyr-AMC (Suc represents succinyl and AMC represents 7-amido-4-methylisocoumarin, obtained from Sigma) for ChT-L activity. 20S proteasome purified from mouse liver $(1 \mu \mathrm{g})$ was incubated with various concentrations of compounds and $50 \mu \mathrm{M}$ fluorogenic peptides in $20 \mathrm{mM}$ Tris- $\mathrm{HCl} \mathrm{pH} 7.8(100 \mu \mathrm{L})$ at $37{ }^{\circ} \mathrm{C}$ for $1 \mathrm{~h}$, respectively. The fluorescence of released AMC was measured by a spectrofluorimeter (Fluostar OPTIMA, BMG Germany) at excitation/emission wavelengths of $380 / 440 \mathrm{~nm}$ and $335 / 410 \mathrm{~nm}$, respectively. $0.1 \%$ DMSO was used as solvent control. Compared with the fluorescence of solvent control, an inhibition rate was calculated and thereafter the $\mathrm{IC}_{50}$ value was deduced. 


\subsection{Molecular Docking}

The covalent docking method with Gold 4.0: A radius of $20 \AA$ from the $\beta 5$-catalytic N-terminal threonine was used to direct site location. For each of the genetic algorithm runs, a maximum number of 100,000 operations were performed on a population of 100 individuals with a selection pressure of 1.1. Operator weights for crossover, mutation, and migration were set to 95, 95, and 10, respectively, as recommended by the authors of the software. 50 GA runs were performed in each docking experiment as done in the software validation procedure. The default GOLD fitness function was used to identify the better binding mode. The distance for hydrogen bonding was set to $2.5 \AA$ and the cut-off value for van der Waals calculation was set to $4 \AA$. Covalent docking was applied and the terminal carbonyl carbon of all the ligands have been bonded to the hydroxyl oxygen of Thr1.

\section{Conclusions}

Based on the binding analysis of proteasome and its inhibitor, a new series of peptide aldehydes was designed and synthesized. Their abilities to inhibit the $20 \mathrm{~S}$ proteasome were assayed and the results show that some compounds have more potency than the positive control MG132. Covalent docking was used to simulate the binding of the peptide aldehyde compounds with 20S, and the docking mode is similar to that of the observed crystal complex and that the P3-postion substitutes are crucial for inhibitor potency. The suggested binding mode provides a potential way to design more potent inhibitors of the $20 \mathrm{~S}$ proteasome.

\section{Acknowledgments}

We thank Groll M. for kindly providing us with the crystal structure of yeast proteasome:MG101 complex. This study was supported by the National Natural Science Foundation of China (No. 20672010).

\section{References}

1. Etlinger, J.D.; Goldberg, A.L. Soluble Atp-dependent prooteolytic system resonsible for degradation of abnormal proteins in reticulocytes. Proc. Natl. Acad. Sci. USA 1977, 74, 54-58.

2. Hershko, A. Lessons from the discovery of the ubiquitin system. Trends Biochem. Sci. 1996, 21, 445-449.

3. Rock, K.L.; Gramm, C.; Rothstein, L.; Clark, K.; Stein, R.; Dick, L.; Hwang, D.; Goldberg, A.L. Inhibitors of the proteasome block the degradation of most cell-proteins and the generation of peptides presented on Mhc Class-I molecules. Cell 1994, 78, 761-771.

4. Osaki, T.; Kimura, T.; Tatemoto, Y.; Lu, D.P.; Yoneda, K.; Yamamoto, T. Diffuse mode of tumor cell invasion and expression of mutant p53 protein but not of p21 protein are correlated with treatment failure in oral carcinomas and their metastatic foci. Oncology 2000, 59, 36-43.

5. Pagano, M.; Tam, S.W.; Theodoras, A.M.; Beerromero, P.; Delsal, G.; Chau, V.; Yew, P.R.; Draetta, G.F.; Rolfe, M. Role of the ubiquitin-proteasome pathway in regulating abundance of the cyclin-dependent kinase inhibitor p27. Science 1995, 269, 682-685. 
6. Alves-Rodrigues, A.; Gregori, L.; Figueiredo-Pereira, M.E. Ubiquitin, cellular inclusions and their role in neurodegeneration. Trends Neurosci. 1998, 21, 516-520.

7. Merforth, S.; Kuehn, L.; Osmers, A.; Dahmann, B. Alteration of 20S proteasome-subtypes and proteasome activator PA28 in skeletal muscle of rat after induction of diabetes mellitus. Int. J. Biochem. Cell Biol. 2003, 35, 740-748.

8. Sun, J.Z.; Nam, S.K.; Lee, C.S.; Li, B.Y.; Coppola, D.; Hamilton, A.D.; Dou, Q.P.; Sebti, S.M. CEP1612, a dipeptidyl proteasome inhibitor, induces p21(WAF1) and p27(KIP1) expression and apoptosis and inhibits the growth of the human lung adenocarcinoma A-549 in nude mice. Cancer Res. 2001, 61, 1280-1284.

9. Kisselev, A.F.; Goldberg, A.L. Proteasome inhibitors: From research tools to drug candidates. Chem. Biol. 2001, 8, 739-758.

10. Groll, M.; Schellenberg, B.; Bachmann, A.S.; Archer, C.R.; Huber, R.; Powell, T.K.; Lindow, S.; Kaiser, M.; Dudler, R. A plant pathogen virulence factor inhibits the eukaryotic proteasome by a novel mechanism. Nature 2008, 452, 755-758.

11. Pirrung, M.C.; Biswas, G.; Ibarra-Rivera, T.R. Total Synthesis of Syringolin A and B. Org. Lett. 2010, 12, 2402-2405.

12. Borissenko, L.; Groll, M. 20S proteasome and its inhibitors: Crystallographic knowledge for drug development. Chem. Rev. 2007, 107, 687-717.

13. Marques, A.J.; Palanimurugan, R.; Matias, A.C.; Ramos, P.C.; Dohmen, R.J. Catalytic mechanism and assembly of the proteasome. Chem. Rev. 2009, 109, 1509-1536.

14. Genin, E.; Reboud-Ravaux, M.; Vidal, J. Proteasome inhibitors: Recent advances and new perspectives in medicinal chemistry. Curr. Top. Med. Chem. 2010, 10, 232-256.

15. Arrigo, A.P.; Tanaka, K.; Goldberg, A.L.; Welch, W.J. Identity of the 19s prosome particle with the large multifunctional protease complex of mammalian-cells. Nature 1988, 331, 192-194.

16. Lowe, J.; Stock, D.; Jap, R.; Zwickl, P.; Baumeister, W.; Huber, R. Crystal-structure of the 20s proteasome from the archaeon T-acidophilum at 3.4-Angstrom resolution. Science 1995, 268, 533-539.

17. Groll, M.; Ditzel, L.; Lowe, J.; Stock, D.; Bochtler, M.; Bartunik, H.D.; Huber, R. Structure of 20S proteasome from yeast at 2.4 angstrom resolution. Nature 1997, 386, 463-471.

18. Unno, M.; Mizushima, T.; Morimoto, Y.; Tomisugi, Y.; Tanaka, K.; Yasuoka, N.; Tsukihara, T. The structure of the mammalian $20 \mathrm{~S}$ proteasome at 2.75 angstrom resolution. Structure 2002, 10, 609-618.

19. Groll, M.; Larionov, O.V.; Huber, R.; de Meijere, A. Inhibitor-binding mode of homobelactosin C to proteasomes: New insights into class I MHC ligand generation. Proc. Natl. Acad. Sci. USA 2006, 103, 4576-4579.

20. Kaiser, M.; Groll, M.; Siciliano, C.; Assfalg-Machleidt, I.; Weyher, E.; Kohno, J.; Milbradt, A.G.; Renner, C.; Huber, R.; Moroder, L. Binding mode of TMC-95A analogues to eukaryotic 20S proteasome. ChemBioChem 2004, 5, 1256-1266.

21. Groll, M.; Gotz, M.; Kaiser, M.; Weyher, E.; Moroder, L. TMC-95-based inhibitor design provides evidence for the catalytic versatility of the proteasom. Chem. Biol. 2006, 13, 607-614. 
22. Clerc, J.; Florea, B.I.; Kraus, M.; Groll, M.; Huber, R.; Bachman, A.; Dudler, R.; Driessen, C.; Overkleeft, H.S.; Kaiser, M.C. Syringolin A selectively labels the 20S proteasome in murine EL4 and wild-type and bortezomib-adapted leukaemic cell lines. ChemBioChem 2009, 10, 2638-2643.

23. Groll, M.; Berkers, C.R.; Ploegh, H.L.; Ovaa, H. Crystal structure of the boronic acid-based proteasome inhibitor bortezomib in complex with the yeast 20S proteasome. Structure 2006, 14, 451-456.

24. Meng, L.; Mohan, R.; Kwok, B.H.; Elofsson, M.; Sin, N.; Crews, C.M. Epoxomicin, a potent and selective proteasome inhibitor, exhibits in vivo anti-inflammatory activity. Proc. Natl. Acad. Sci. USA 1999, 96, 10403-10408.

25. Palmer, J.T.; Rasnick, D.; Klaus, J.L.; Bromme, D. Vinyl sulfones as mechanism-based cysteine protease inhbibitors. J. Med. Chem. 1995, 38, 3193-3196.

26. Omura, S.; Matsuzaki, K.; Fujimoto, T.; Kosuge, K.; Furuya, T.; Fujita, S.; Nakagawa, A. Structure of lactacystin, a new microbial metabolite which induces differentiation of neuroblastoma cells. J. Antibiot. 1991, 44, 117-118.

27. Fenteany, G.; Standaert, R.F.; Lane, W.S.; Chois, S.; Corey, E.J.; Schreiber, S.L. Inhibition of proteasome activites and subunit-specific amino-terminal threonine modification by lactacystin. Scinece 1995, 268, 726-731.

28. Feling, R.H.; Buchanan, G.O.; Mincer, T.J.; Kauffman, C.A.; Jensen, P.R.; Fenical, W. Salinosporamide A: A highly cytotoxic proteasome inhibitor from a novel microbial source, a marine bacterium of the new genus salinospora. Angew. Chem. Int. Ed. 2003, 42, 355-357.

29. Groll, M.; Huber, R.; Potts, B.C.M. Crystal structures of salinosporamide A (NPI-0052) and B (NPI-0047) in complex with the 20S proteasome reveal important consequences of beta-lactone ring opening and a mechanism for irreversible binding. J. Am. Chem. Soc. 2006, 128, 5136-5141.

30. Vinitsky, A.; Michaud, C.; Powers, J.C.; Orlowski, M. Inhibition of the chymotrypsin-Like activity of the pituitary multicatalytic proteinase complex. Biochemistry 1992, 31, 9421-9428.

31. Momose, I.; Umezawa, Y.; Hirosawa, S.; Iinuma, H.; Ikeda, D. Structure-based design of derivatives of tyropeptin A as the potent and selective inhibitors of mammalian 20S proteasome. Bioorg. Med. Chem. Lett. 2005, 15, 1867-1871.

32. Loidl, G.; Groll, M.; Musiol, H.J.; Ditzel, L.; Huber, R.; Moroder, L. Bifunctional inhibitors of the trypsin-like activity of eukaryotic proteasomes. Chem. Biol. 1999, 6, 197-204.

33. Ma, Y.; Chen, B.; Liu, D.; Yang, Y.; Xiong, Z.; Zeng, J.; Dong, Y. MG132 treatment attenuates cardiac remodeling and dysfunction followingaortic banding in rats via the NF- $\mathrm{B} / \mathrm{TGF} \beta 1$ pathway. Biochem. Pharmacol. 2011, 81, 1228-1236.

34. Donkor, I.O. A survey of calpain inhibitors. Curr. Med. Chem. 2000, 7, 1171-1188.

35. Tsubuki, S.; Saito, Y.; Tomioka, M.; Ito, H.; Kawashima, S. Differential inhibition of calpain and proteasome activities by peptidyl aldehydes of di-leucine and tri-leucine. J. Biochem. 1996, 119, 572-576.

36. Zhang, X.C.; Kumar, S.; Chen, J.H.; Teplyakov, A.V. Covalent attachment of shape-restricted DNA molecules on amine-functionalized Si(III) surface. Surface Sci. 2009, 603, 2445-2457.

37. Zhu, Y.Q.; Pei, J.F.; Liu, Z.M.; Lai, L.H.; Cui, J.R.; Li, R.T. 3D-QSAR studies on tripeptide aldehyde inhibitors of proteasome using CoMFA and CoMSIA methods. Bioorg. Med. Chem. 2006, 14, 1483-1496. 
38. Liu, J.; Zhang, H.; Xiao, Z.; Wang, F.; Wang, X.; Wang, Y. Combined 3D-QSAR, molecular focking and molecular fynamics dtudy on ferivatives of peptide epoxyketone and tyropeptinboronic acid as inhibitors against the $\beta 5$ subunit of Human 20S proteasome. Int. J. Mol. Sci. 2011, $12,1807-1835$.

39. Mckennon, M.J.; Meyers, A.I.; Drauz, K.; Schwarm, M. A Convenient reduction of amino-acids and their derivatives. J. Org. Chem. 1993, 58, 3568-3571.

40. Drag, M.; Latajka, R.; Gumienna-Kontecka, E.; Kozlowski, H.; Kafarski, P. Stereoselective synthesis, solution structure and metal complexes of (1S,2S)-2-amino-1-hydroxyalkylphosphonic acids. Tetrahedron Asymmetry 2003, 14, 1837-1845.

41. Fu, Y.Q.; Xu, B.; Zou, X.M.; Ma, C.; Yang, X.M.; Mou, K.; Fu, G.; Lu, Y.; Xu, P. Design and synthesis of a novel class of furan-based molecules as potential $20 \mathrm{~S}$ proteasome inhibitors. Bioorg. Med. Chem. Lett. 2007, 17, 1102-1106.

42. Zhu, Y.Q.; Zhao, X.; Zhu, X.R.; Wu, G.; Li, Y.J.; Ma, Y.H.; Yuan, Y.X.; Yang, J.; Hu, Y.; Ai, L.; Gao, Q.Z. Design, synthesis, biological evaluation, and structure-activity relationship (SAR) discussion of dipeptidyl boronate proteasome inhibitors, Part I: Comprehensive understanding of the SAR of $\alpha$-amino acid boronates. J. Med. Chem. 2009, 52, 4192-4199.

43. Rydzewski, R.M.; Burrill, L.; Mendonca, R.; Palmer, J.T.; Rice, M.; Tahilramani, R.; Bass, K.E.; Leung, L.; Gjerstad, E.; Janc, J.W.; Pan, L. Optimization of subsite binding to the 5 subunit of the human 20S proteasome using vinyl sulfones and 2-keto-1, 3, 4-oxadiazoles: Syntheses and cellular properties of potent, selective proteasome inhibitors. J. Med. Chem. 2006, 49, 2953-296.

Sample Availability: Samples of the compounds 3a-3r are available from the authors.

(C) 2011 by the authors; licensee MDPI, Basel, Switzerland. This article is an open access article distributed under the terms and conditions of the Creative Commons Attribution license (http://creativecommons.org/licenses/by/3.0/). 